\title{
La vulnerabilidad urbana en España. Identificación y evolución de los barrios vulnerables
}

\author{
The urban vulnerability in Spain. Identification and evolution \\ of the vulnerable neighborhoods
}

\author{
Julio Alguacil Gómez y Javier Camacho Gutiérrez \\ Universidad Carlos III \\ Agustín Hernández Aja \\ Universidad Politécnica de Madrid \\ jalguaci@polsoc.uc3m.es (ESPAÑA)
}

Recibido: 9.102012

Aceptado: 5.10.2013

\section{RESUMEN}

La realidad de nuestras ciudades muestra un mosaico de áreas diversas articuladas por las redes de transporte y clasificadas según niveles de renta. Los patrones básicos del mapa son fijos: existen áreas que se constituyeron desde sus inicios para acoger a una población u otra, mientras que otras zonas fueron abandonadas por sus poblaciones iniciales (como los centros históricos) y ocupan en estos momentos un lugar inferior en el rango urbano. La suma de las demandas sociales y políticas de redistribución hicieron que el planeamiento urbano (en la mejor de sus identidades) se conformase como una herramienta para el desarrollo de un proyecto en pro del «derecho a la ciudad», restañando rupturas espaciales y resolviendo las carencias de dotaciones y accesos necesarios para que la totalidad de los espacios fuese parte integral de la ciudad, de forma que sus habitantes pudiesen ejercer la condición de ciudadanía. Pero la propia biografía de la ciudad desarrolla en paralelo una reordenación continua de sus espacios, de forma que el proyecto igualitario que garantiza la condición de ciudadanía es interceptado por el proyecto inmobiliario, que busca mediante la segmentación espacial y la reubicación de sus habitantes en función de sus rentas, el máximo beneficio.

EMPIRIA. Revista de Metodología de Ciencias Sociales. N. . 27, enero-abril, 2014, pp. 73-94. ISSN: 1139-5737 DOI 10.5944/empiria.27.10863 
Si apostamos por un urbanismo que busque asegurar el «derecho a la ciudad», éste debe basarse en un proyecto de constante reconstrucción de los equilibrios entre las áreas que el mercado tiende a dividir y segmentar. Es necesario por tanto disponer del mapa de las áreas que han perdido o están en riesgo de perder su puesto en la ciudad, a estas áreas las denominamos aquí Barrios Vulnerables y presentamos un método para su determinación.

\section{PALABRAS CLAVE}

Vulnerabilidad Urbana, Cohesión Social, Espacio Urbano, Delimitación Espacial, Planificación Integral

\section{SUMMARY}

The reality of our cities shows a mosaic of different articulated by transport networks and classified according income levels. The fixed basic patterns that characterize the urban map are areas that were established from the outset to accommodate a population or another and other areas that were abandoned by their initial populations (as historical centers) and at this moment occupy a lower rank in urban segmentation. The amount of social demands and redistribution policies made conform to urban planning (in the best of their identities) as a tool for the development of a project in favor of the «right to the city», avoiding spatial ruptures, and solving the shortcomings necessary equipments and accesses, so that all the spaces were integrated in the city, so that its inhabitants could exercise citizenship status. But the biography of the city itself, developed in parallel, a continuous rearrangement of spaces, so the project that guarantees equal citizenship status is intercepted by the building project that seeks through spatial segmentation and the relocation of its inhabitants according to their maximum benefit income.

If we focus on urban planning to seek and to ensure the «right to the city», it must be based on an ongoing project for rebuilding the balance between areas that the market tends to divide and segment. It is therefore necessary to have the map of those areas that have lost or are at risk of losing their place in the city, we call these areas Vulnerable Neighborhoods and we present a method for its determination.

\section{KEYWORDS}

Urban Vulnerability, Social Cohesion, Urban Space, Space Delimitation, Integrated Planning

EMPIRIA. Revista de Metodología de Ciencias Sociales. N. ${ }^{2}$ 27, enero-abril, 2014, pp. 73-94. ISSN: 1139-5737 DOI 10.5944/empiria.27.10863 


\section{INTRODUCCIÓN}

La metodología para el estudio de la vulnerabilidad urbana que se presenta en este artículo sirvió para la elaboración del Catálogo de Barrios Vulnerables incluido en el Observatorio de la Vulnerabilidad Urbana del Ministerio de Fomento (http://siu.vivienda.es/portal/index.php?option=com_content\&view=ar ticle\&id=82\&Itemid=75\&lang=es ) puesto en marcha por la Dirección General de Suelo y Políticas Urbanas del Ministerio de Fomento, en colaboración con el Departamento de Urbanística y Ordenación del Territorio (DUyOT) de la Universidad Politécnica de Madrid (UPM). El trabajo retoma en 2009 el primer catálogo realizado en 1996, también en colaboración con el DUyOT (Hernández, A. 1997), que fue recogido en el pionero trabajo: «La desigualdad urbana en España» (Arias 2000); y es fruto del trabajo realizado por un equipo multidisciplinar, que se formó bajo el paraguas de dicha colaboración institucional ${ }^{1}$.

El objetivo básico del análisis era la identificación, delimitación y análisis de la vulnerabilidad urbana en los barrios de aquellas ciudades de más de 50.000 habitantes y las capitales de provincia que no llegan a esa cifra. El resultado es un catálogo con vocación operativa, en la medida en que puede ser útil para diseñar procesos de intervención en estas áreas urbanas, para paliar su situación de vulnerabilidad. Además, la herramienta de análisis cobra un mayor sentido al adoptar una estrategia diacrónica, puesto que el catálogo de barrios vulnerables se desglosa en tres, correspondientes a los años 1991, 2001 y 2006. Así, el artículo hace especial hincapié en la definición del concepto de «vulnerabilidad», en la metodología utilizada para su identificación y caracterización y en la comparación de la evolución de la vulnerabilidad en las áreas urbanas españolas.

\footnotetext{
1 Director del trabajo:

- Agustín Hernández Aja, arquitecto, director del Departamento de Urbanística y Ordenación del Territorio de la ETSAM.

Redactores:

- Agustín Hernández Aja, arquitecto, director del Departamento de Urbanística y Ordenación del Territorio de la ETSAM.

- Mariano Vázquez Espí, arquitecto y profesor titular del Departamento de Estructuras de edificación.

- Carolina García Madruga, arquitecta y alumna del Master de Planeamiento Urbano y de doctorado.

- Ángela Matesanz Parellada, arquitecta y alumna del Master de Planeamiento Urbano y de doctorado.

- Elena Moreno García, arquitecta y alumna del Master de Planeamiento Urbano.

- Julio Alguacil, sociólogo y profesor de la Universidad Carlos III.

- Javier Camacho, sociólogo y profesor de la Universidad Carlos III.

Comisión de Seguimiento del Ministerio de Fomento.

- Ángela de la Cruz Mera, Subdirectora General de Urbanismo. Urbanismo.

- José Luis Nicolás Rodrigo, sociólogo, Consejero Técnico de la Subdirección General de

- Eduardo de Santiago Rodríguez, arquitecto, Consejero Técnico de Suelo y Políticas Urbanas. Secretaría General de Vivienda.

Asesoría del Ministerio de Fomento: ARGEA Consultores SL.
}

EMPIRIA. Revista de Metodología de Ciencias Sociales. N. ${ }^{2}$ 27, enero-abril, 2014, pp. 73-94. ISSN: 1139-5737 DOI 10.5944/empiria.27.10863 


\section{EL CONCEPTO DE VULNERABILIDAD}

«vulnerable (del lat. «vulnerabilis») adj. Susceptible de ser herido o vulnerado, en cualquier acepción, de recibir un daño o perjuicio, o de ser afectado, conmovido, convencido o vencido por algo (...)» (Moliner 2008: 528).

En su sentido etimológico, el término vulnerable expresa la «susceptibilidad»o posibilidad de ser herido, de recibir un daño o de ser afectado por alguna circunstancia.

El Departamento de Asuntos Económicos y Sociales de las Naciones Unidas ofrece la siguiente definición del concepto «vulnerabilidad»:

«(...) En esencia, la vulnerabilidad puede definirse como un estado de elevada exposición a determinados riesgos e incertidumbres, combinado con una capacidad disminuida para protegerse o defenderse de ellos y hacer frente a sus consecuencias negativas. La vulnerabilidad existe en todos los niveles y dimensiones de la sociedad y es parte integrante de la condición humana, por lo que afecta tanto a cada persona como a la sociedad en su totalidad».

(Naciones Unidas 2003: 8)

Es un concepto, por tanto, que acoge múltiples dimensiones que interactúan entre sí, y que en su vertiente social, inaugurada por Robert Castel (1991, 1995), contrasta con la visión de «los excluidos» ofrecida por René Lenoir ${ }^{2}$ y acogida por las políticas sociales del gobierno francés en la década de los 80. La exclusión no es entendida por Lenoir tanto como un proceso, sino como un estado de marginación adscrito a las condiciones de los sujetos. La propuesta de $\mathrm{Castel}^{3}$ consistente en establecer una zona social intermedia entre la zona de exclusión (o desafiliación) y la zona de integración, refuerza la idea sobre el carácter dinámico de la exclusión social, precisamente al identificar con solidez una zona de vulnerabilidad

2 Alto funcionario francés y asesor directo del presidente Chirac para las cuestiones sociales, se le suele considerar el autor que incorpora el concepto de exclusión social.

3 La propuesta de Castel, que acogemos en la sugerencia del propio Castel para su aplicación al análisis del hecho urbano, la sintetiza de la siguiente manera: «He aquí entonces un modelo que en un principio quizá parecerá demasiado teórico, pero del que espero, sin embargo, demostrar la capacidad para generar implicaciones prácticas interesantes.

Propongo ubicar las situaciones marginales al final de un doble proceso: de desenganche en relación al trabajo y en relación a la inserción relacional. Advertimos enseguida que todo individuo puede situarse en relación a ese doble eje de la integración a través del trabajo y de la inscripción relacional. Esquematizando mucho distingamos tres valores en cada uno de los ejes: trabajo estable-trabajo precario-no trabajo e inserción relacional fuerte fragilidad relacional-aislamiento social. Acoplando esos valores de dos en dos se obtienen tres zonas, a saber la zona de integración (trabajo estable y fuerte inscripción relacional, que a menudo van juntos), la zona de vulnerabilidad (trabajo precario y fragilidad de los soportes relacionales), y la zona de marginalidad, que prefiero llamar zona de desafiliación para marcar bien la amplitud del doble desenganche: ausencia de trabajo y aislamiento relacional (Castel, 1991: 41). El propio Castel amplía su modelo a una cuarta zona intermedia, «zona de asistencia» que puede considerarse una variante de la zona de vulnerabilidad: «El tratamiento de la indigencia inválida representa así una cuarta zona, la zona de asistencia. Esta cumple con una protección cercana sobre la base del «domicilio de emergencia». La mayor o menor presencia de las políticas sociales o asistenciales determinan la mayor o menor presencia de atributos de vulnerabilidad.

EMPIRIA. Revista de Metodología de Ciencias Sociales. N. ${ }^{\circ}$ 27, enero-abril, 2014, pp. 73-94. ISSN: 1139-5737 DOI 10.5944/empiria.27.10863 
que es la antesala de la exclusión. Esta zona de vulnerabilidad la identifica Robert Castel como «[...] una zona intermediaria, inestable, que conjuga la precariedad del trabajo y la fragilidad de los soportes relacionales» (Castel 1995: 17). Considerada como zona de tránsito entre la zona de integración y de exclusión estos atributos pueden transferirse al territorio, combinándose con nuevas dimensiones, y así identificar lugares (en nuestro caso barrios) donde se produce la intersección de fragilidades entre el trabajo y las relaciones sociales y que sitúa a los sujetos y grupos en riesgo de caer hacia la zona de exclusión social.

En términos urbanos, y aplicada sobre un espacio social considerado como lugar geográfico, la «vulnerabilidad urbana» se referiría a la potencialidad de que la población de un determinado espacio urbano concreto sea afectada por alguna(s) circunstancia(s) adversa(s), de modo que el concepto alude no tanto a la existencia de una situación crítica constatada en la actualidad como a la de unas determinadas condiciones de riesgo, fragilidad y desventaja que harían posible la entrada en esa situación crítica de desfavorecimiento, entendido éste como «la materialización de dicho riesgo en una situación de exclusión ya consolidada» (Bruquetas, Moreno, Walliser 2005: 11). Respecto del concepto de exclusión social recoge, poniéndolas en relación, tanto la polarización propia del eje vertical (arriba/abajo; riqueza/pobreza) como la segmentación definida en un eje horizontal (dentro/fuera) señalando las distancias entre los que participan del sistema y los que quedan fuera de él. En el sistema urbano, hablaríamos de segregación espacial cuando hay barrios que quedan fuera del sistema urbano «normalizado» (EDIS 2000) y por lo tanto en una situación de vulnerabilidad.

De este modo, el concepto estaría íntimamente relacionado con un punto de vista operativo, es decir, con la aplicación de actuaciones o medidas preventivas ${ }^{4}$ para que las potencialidades negativas no se conviertan finalmente en hechos, «de forma que de no actuarse sobre las bases del problema el área entrará en crisis, pudiéndose producir una degradación funcional y social del ámbito que lo conduzca a la marginación» (Hernández Aja 2007: 5) Por el contrario, el término «área problema» induce a pensar que el espacio delimitado tan sólo tiene carencias materiales, que podrían revertirse mediante medidas de ampliación o renovación de las dotaciones existentes. Así, mientras el término «vulnerable» indica la necesidad de acciones integrales sobre un área, el término problema permitiría colegir que la solución a éste podría pasar simplemente por el desarrollo y ampliación de las políticas sectoriales al uso.

Profundizando en el mismo sentido, puede entenderse por vulnerabilidad urbana «como aquel proceso de malestar en las ciudades producido por la combinación de múltiples dimensiones de desventaja, en el que toda esperanza de movilidad social ascendente, de superación de su condición social de exclusión o próxima a ella, es contemplada como extremadamente difícil de alcanzar. Por el contrario, conlleva una percepción de inseguridad y miedo a la posibilidad de una movilidad social descendente, de empeoramiento de sus actuales condiciones de vida» (Alguacil 2006: 161).

\footnotetext{
4 Frente a la que podrían ser otros puntos de vista de tipo «curativo» $\mathbf{0}$ «paliativo».
} 
Hay que insistir, a la vista de dicha definición, que el concepto de vulnerabilidad hace referencia a dos cuestiones:

- El incremento de las amenazas y los riesgos que afectan a las personas/ sociedades/grupos sociales/estados.

- El debilitamiento de los mecanismos para afrontar dichos riesgos y amenazas.

Con esas consideraciones, es evidente que, en las últimas décadas, tal y como muestra Castel $(1991,1995)$, la exposición de la población a esas amenazas ha aumentado, mientras que se ha producido simultáneamente un debilitamiento de los mecanismos para afrontarlos. La importancia o el grado de adecuación del concepto, desde la perspectiva del análisis socio-urbanístico, muestran que para comprender las raíces del orden social y del bienestar humano se requiere de un sentido de pertenencia a la sociedad, de modo que se satisfagan las necesidades materiales, culturales, psíquicas, relacionales, etc. como mecanismos de inclusión.

En consecuencia, el concepto de vulnerabilidad social media entre la inclusión y la exclusión, siendo la antesala de ésta, expresando una problemática compleja que, para abordarla, precisa de un análisis y de una intervención multidimensional, contemplando aquellas dimensiones y variables que más incidencia tienen en la disminución o crecimiento de la vulnerabilidad en un espacio social concreto.

La vulnerabilidad urbana deriva de dos factores principales desencadenantes: la exclusión residencial y la exclusión laboral, que en su combinación se refuerzan mutuamente y desencadenan otros factores inhibidores, de carácter político, cultural, social, personal...

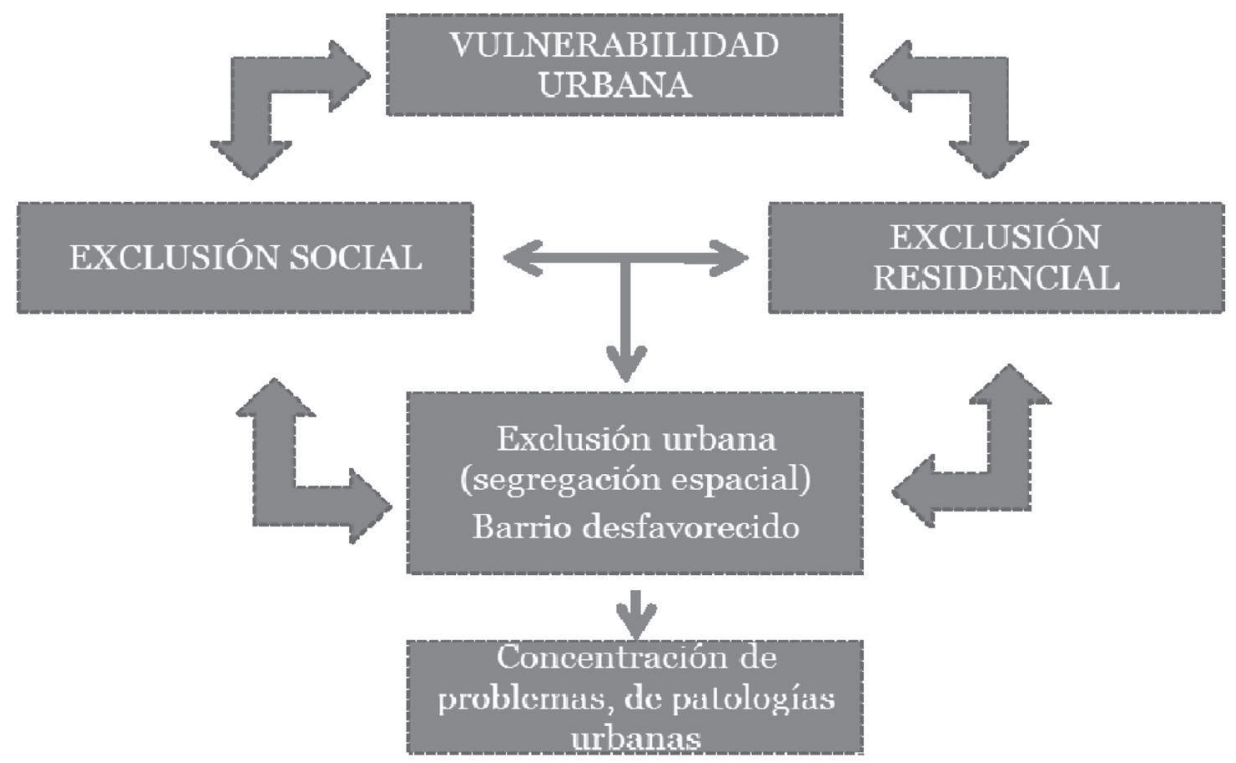

Fuente: VV.AA. (2013): La vivienda en España en el siglo XXI. Fundación FOESSA, pp. 318.

EMPIRIA. Revista de Metodología de Ciencias Sociales. N. ${ }^{2}$ 27, enero-abril, 2014, pp. 73-94. ISSN: 1139-5737 DOI 10.5944/empiria.27.10863 
Cuando estas situaciones se concentran en determinados lugares, por los efectos de la segregación espacial, se suelen reforzar e ir acompañadas de un medio social y económicamente decaído y un medio ambiente urbano degradado. En estos casos, no sólo la exclusión residencial y social se combinan y se retroalimentan, sino que estas condiciones se reubican recurrentemente en el propio medio, generando un proceso circular que reproduce sus componentes.

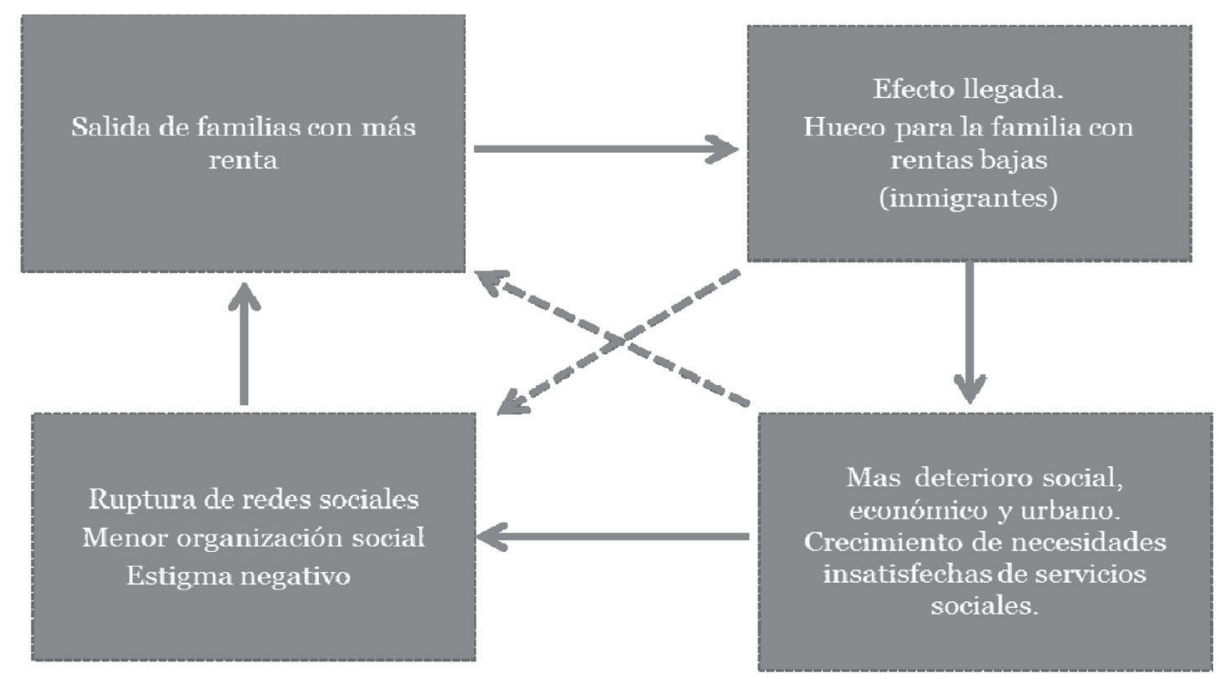

Fuente: elaboración propia.

De este modo, la vulnerabilidad de un territorio combina factores objetivos y factores subjetivos:

- Por un lado, está constituida por condiciones de desfavorecimiento social, de desventajas estructurales de una población para desarrollar proyectos vitales en contextos de seguridad y confianza.

- Por otro lado, la vulnerabilidad es también un entorno psicosocial que afecta a la percepción que los ciudadanos tienen del territorio en donde viven y de sus propias condiciones sociales. Una percepción negativa de su situación como residentes en un espacio social puede traducirse en procesos de malestar urbano, que pueden no corresponderse con unos indicadores «objetivos» de vulnerabilidad. Estamos hablando, por tanto, de que la vulnerabilidad es también un concepto relativo, contextual, que debe ser enmarcado en un territorio concreto.

Esa complejidad del concepto debe trasladarse a los instrumentos para medirla. La disponibilidad de datos estadísticos actualizados es un elemento central para el análisis sociológico, pero encuentra serias dificultades, sobre todo para

EMPIRIA. Revista de Metodología de Ciencias Sociales. N. ${ }^{2}$ 27, enero-abril, 2014, pp. 73-94. ISSN: 1139-5737 DOI 10.5944/empiria.27.10863 
escalas de análisis inferiores al nivel municipal, y más concretamente al nivel de sección censal, que es el nivel imprescindible para abordar un análisis espacial detallado. Dado que la pretensión es construir un concepto de vulnerabilidad multidimensional, la medición de la misma debe hacerse a través de indicadores diversos que expresen la complejidad al combinar diferentes dimensiones, y ello nos enfrenta con el problema de las fuentes: sólo es posible utilizar el Censo de Población y Vivienda en la obtención del conjunto de datos necesarios a nivel inframunicipal, pues aunque para algunas variables socio-demográficas sí existe la posibilidad de acceder a los datos anuales del Padrón Municipal de Habitantes, dicha información estadística es insuficiente para elaborar una actualización intermedia completa entre los períodos censales. Por lo tanto, hemos de limitarnos - hasta que no estén disponibles los resultados del Censo de Población y Vivienda 2011 - al análisis de los datos estadísticos contenidos en el Censo de 2001. A través de dicha fuente, y tomando como primera referencia el estudio realizado para el año 1991, será posible establecer un análisis de la evolución de la vulnerabilidad en las áreas delimitadas a través de las secciones censales.

Una vez expuestas las limitaciones metodológicas de las fuentes estadísticas disponibles, la primera pregunta que debemos hacernos es cómo mediremos la vulnerabilidad de estas áreas homogéneas. Para ello, el punto de partida debe ser el carácter multidimensional de la vulnerabilidad social antes mencionada y para ello partimos de la identificación de cuatro ejes básicos.

\subsection{La vulnerabilidad socio-demográfica}

En la sociedad española actual se aprecian tres fenómenos demográficos que tienen serias implicaciones en el aumento de la vulnerabilidad social:

- El envejecimiento demográfico: los procesos de envejecimiento demográfico son característicos de las sociedades occidentales desarrolladas, aspecto que tiene serias implicaciones sociales, puesto que reduce la base demográfica productiva e incrementa el porcentaje de población dependiente. Las necesidades que afectan a esta población se aprecian en múltiples demandas que deben ser atendidas por los poderes públicos, que no siempre ofrecen una respuesta adecuada a dichas necesidades. La dependencia, los problemas sanitarios, el deterioro de sus viviendas y la escasez de rentas derivadas de pensiones de jubilación modestas, pueden ser factores que conduzcan a su consideración como población vulnerable. La creciente presencia de hogares unipersonales constituidos por personas mayores es un aspecto social de la mayor importancia, puesto que la soledad de estas personas, sus dificultades de movilidad y sus necesidades sanitarias, relacionales, afectivas, etc., deben constituir objeto de atención para los servicios sociales municipales, en especial, a través de la ayuda a domicilio y otras iniciativas que pretenden ayudar a los ancianos que viven en soledad. 
- La complejización de la estructura de los hogares: con la aparición y crecimiento de tipologías que presentan un grado mayor de vulnerabilidad ante el debilitamiento de las redes sociales y familiares de ayuda: por ejemplo, destacan por su especial vulnerabilidad los hogares monoparentales, pues a la necesidad de trabajar de la persona de referencia de dichos hogares, se une las dificultades para atender de forma adecuada las necesidades afectivas, materiales, formativas, etc., de los hijos a su cargo. Desde ese mismo enfoque de la vulnerabilidad de los hogares, también están aumentando los hogares unipersonales, a consecuencia del envejecimiento demográfico (viudas en soledad, personas mayores dependientes sin ayuda...). Por último, y aunque es un fenómeno difícil de medir pues en muchas ocasiones no está suficientemente registrado en el Padrón, los hogares extensos y múltiples pueden estar incrementando su peso proporcional a consecuencia de la llegada de población extranjera inmigrante que, como único medio para afrontar los gastos derivados del alquiler o compra de la vivienda, deben recurrir a compartir el alojamiento con más personas para abaratar el coste: el hacinamiento residencial sería la consecuencia de esta necesidad.

- El «boom» de la inmigración extranjera proveniente de países no desarrollados: la llegada de inmigrantes extranjeros es un fenómeno que puede contribuir a limitar el proceso de envejecimiento demográfico. Sin embargo, debe producirse una política específica que favorezca la cohesión e integración social de estos colectivos en la sociedad de acogida. En ese sentido, es una población potencialmente vulnerable, en la medida en que se trata, en su mayoría, de personas procedentes de países en vías de desarrollo que no encuentran en sus lugares de origen la posibilidad de salir adelante y que ven en el primer mundo una oportunidad para conseguir una vida más digna y un futuro para sí y sus hijos. Sin embargo, esas oportunidades no siempre se les ofrecen, pues su posición en el mercado laboral es de tal precariedad que implica un mayor impacto en sus tasas de desempleo y en sus modestos niveles de renta.

Los inmigrantes extranjeros llegan a un país desconocido, produciéndose un choque socio-cultural que genera fricciones entre los distintos grupos nacionales y étnicos. Además de los problemas de convivencia que se producen, la población inmigrante necesita un apoyo en materias tales como la formación, la inserción laboral, el acceso a la vivienda, la ayuda de los servicios sociales, etc. Por lo tanto, los servicios públicos se enfrentan a una nueva situación, con la aparición de nuevos usuarios que presentan distintas necesidades y que tienen unas características particulares que hacen necesaria una adecuación de la intervención social. Por lo tanto, contextos donde la inmigración crece de forma notable en pocos años, pueden conformar espacios de vulnerabilidad, cuando no de exclusión y marginación social, sino se realizan esfuerzos de integración social.

EMPIRIA. Revista de Metodología de Ciencias Sociales. N. ${ }^{\circ}$ 27, enero-abril, 2014, pp. 73-94. ISSN: 1139-5737 DOI 10.5944/empiria.27.10863 


\subsection{La vulnerabilidad socio-económica}

Tiene que ver con la capacidad de los hogares para procurarse de manera satisfactoria un cierto bienestar material y emocional. La existencia de empleo con unas ciertas condiciones de estabilidad y salario, es la principal fuente de acceso a ese bienestar material y emocional: el desempleo y una excesiva precariedad en el empleo serían factores que dificultarían ese objetivo, que a su vez está muy relacionado con los niveles formativos de la población, que permiten acceder a un mercado laboral primario (definido por mejores condiciones laborales y salariales) o secundario (definido por la precariedad). Por lo tanto, los tres fenómenos determinantes para focalizar el problema de la vulnerabilidad socioeconómica tienen que ver con tres variables básicas:

- El desempleo: en un periodo de crisis como el actual, asistimos con preocupación a unas elevadas tasas de desempleo, una característica estructural de nuestro mercado laboral, que no es capaz de incorporar a la población activa en su totalidad, manteniendo un diferencial apreciable en relación a otros países de nuestro entorno.

- La precariedad laboral: el «milagro económico español» de los últimos años se ha sustentado sobre una base productiva que generaba un empleo precario, en gran parte de escasa cualificación. Esa precariedad supone, en primer lugar, el peligro de desembocar en una situación de desempleo, sobre todo en periodos de crisis como el actual y, en segundo lugar, la aparición y consolidación de una población trabajadora con un nivel salarial que no asegura ya la cobertura de las necesidades básicas de sus familias. Es decir, existe una capa notable de trabajadores con bajo salario crónico que constituyen ya un grupo incorporado al colectivo de la pobreza.

- Los bajos niveles formativos de las clases trabajadoras: que dificultan extraordinariamente los procesos de movilidad social ascendente y reproducen las desigualdades sociales. En especial, hay que destacar los elevados niveles de fracaso y abandono escolar que afecta a los jóvenes de estas fracciones sociales y cómo ese factor es determinante para entender las elevadas tasas de desempleo y de precariedad laboral existentes entre dichos colectivos.

\subsection{La vulnerabilidad residencial}

Los soportes físicos que acogen la vida de los ciudadanos son muy importantes, puesto que sus malas condiciones no permitirán un desarrollo satisfactorio de la vida cotidiana: una dimensión básica de la condición humana es la adecuación del hábitat a las necesidades de la población. El factor residencial no hace referencia exclusivamente a las viviendas donde se desarrolla la esfera privada de nuestras vidas. Por eso, en lugar de vivienda, el concepto de alojamiento

EMPIRIA. Revista de Metodología de Ciencias Sociales. N. ${ }^{\circ}$ 27, enero-abril, 2014, pp. 73-94. ISSN: 1139-5737 DOI 10.5944/empiria.27.10863 
remite a una dimensión más compleja, que hace referencia tanto a las viviendas como al entorno en el que se desarrolla buena parte de nuestra vida social: el hombre, en cuanto ser social, necesita de la dimensión relacional para ser completo. En ese sentido, un hábitat degradado, tanto si afecta a las viviendas como al entorno vecinal, son expresiones de condiciones desfavorables que pueden propiciar la aparición de sentimientos de vulnerabilidad. La infravivienda sería la máxima expresión de la vulnerabilidad residencial, entendida como aquellos alojamientos que no reúnen condiciones dignas de habitabilidad, ya sea por el mal estado de conservación de los edificios, por disponer de una superficie insuficiente de acuerdo a los residentes que habitan la vivienda o bien por la falta de instalaciones básicas en la vivienda (calefacción, aseo/servicio, ascensor,...).

\subsection{La vulnerabilidad subjetiva}

Otro factor a considerar son las percepciones subjetivas que tienen los residentes respecto del medio urbano y social donde se ubica su residencia y que se puede considerar como referencia de la calidad del entorno residencial (vecindario, barrio). Ello es importante porque la opinión de las personas sobre su entorno residencial y habitacional expresa la existencia o no de cierto malestar urbano, indicador de una serie de circunstancias que inciden en el grado de vulnerabilidad social de sus residentes.

El Censo de Población y Vivienda de 2001 incorpora, respecto de censos anteriores, algunas innovaciones de interés. Se desarrolla un bloque sobre la percepción que las personas de referencia de cada hogar tienen sobre el entorno residencial. Bajo la pregunta « ¿Tiene su vivienda alguno de los problemas siguientes?» se despliegan ítems que permiten construir indicadores basados en las percepciones subjetivas de la realidad del medio social y ambiental de la vivienda de referencia a través de preguntas dirigidas a conocer el grado de satisfacción de los hogares con las viviendas que habitan y las zonas en que se encuentra:

- Ruidos exteriores.

- Contaminación o malos olores provocados por la industria, el tráfico...

- Malas comunicaciones.

- Pocas zonas verdes (parques, jardines...).

- Delincuencia o vandalismo en la zona.

\section{LA METODOLOGÍA EMPLEADA EN EL ANÁLISIS DE LA VULNERABILIDAD URBANA}

El estudio de la vulnerabilidad en barrios o áreas homogéneas del territorio nacional intenta dar continuidad al trabajo realizado por el Ministerio de

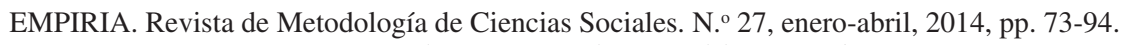
ISSN: 1139-5737 DOI 10.5944/empiria.27.10863 
Fomento, el INE y el Instituto Juan de Herrera en el año 1996 (Hernández, A. 1997) que identificó y delimitó unas áreas vulnerables en el ámbito urbano del Estado Español. Los datos utilizados en ese informe correspondían a información obtenida del Censo de Población y Vivienda del año 1991. Evidentemente, a lo largo de estos años, se han producido importantes cambios sociales en nuestro país, por lo que es preciso revisar el estudio para observar, tanto la evolución de los barrios vulnerables que en su día se identificaron, como la posible existencia de otros nuevos. En ese sentido, es el Censo de Población y Vivienda del año 2001 el que permite un análisis comparativo exhaustivo, teniendo en cuenta que se precisa obtener información a escala de sección censal.

El trabajo desarrollado se ha estructurado en tres fases de análisis:

\subsection{Primera fase: identificación de áreas vulnerables}

Uno de los objetivos principales del estudio de la vulnerabilidad es construir un Observatorio que permita una continuidad en su seguimiento y análisis, que sólo es posible si se trabaja con datos que puedan ser actualizados en el tiempo. Es por ello, que se optó por la selección de los mismos indicadores utilizados en el anterior estudio (Hernández, A. 1997) para la definición de dichas áreas.

La identificación de las áreas vulnerables se ha realizado a través de la agrupación de secciones censales colindantes de cierta homogeneidad urbanística, con un tamaño demográfico entre los 3.500 y los 15.000 habitantes, en las que al menos se contara con una incidencia significativa de uno o más de los tres Indicadores de Vulnerabilidad considerados. Estos tres indicadores son los siguientes:

- Tasa de paro: Según la definición del INE, la tasa de paro para personas residentes en viviendas familiares es el «Porcentaje de la población de 16 años o más en situación de paro respecto al total de la población activa de 16 años o más» 5

La vulnerabilidad se establece cuando el indicador es superior en un $50 \%$ a la media nacional. El valor medio nacional era en 2001 de 14,16\%, determinándose como índice de vulnerabilidad una tasa del 21,24\%.

5 Una persona está en situación de paro si está simultáneamente:

1. Sin trabajo, es decir, no tiene un empleo por cuenta ajena o por cuenta propia,

2. En busca de trabajo, es decir, ha tomado medidas concretas para buscar un trabajo por cuenta ajena o ha hecho gestiones para establecerse por su cuenta (inscripciones en oficinas de paro, gestiones en lugares de trabajo, respuesta a anuncios de periódico, etc.), y

3. Disponible para trabajar en un empleo por cuenta ajena o propia. Se considera población económicamente activa a todas las personas de 16 o más años que satisfacen las condiciones necesarias para su inclusión entre las personas ocupadas o paradas. Una persona está ocupada si durante la semana de referencia ha tenido un trabajo por cuenta ajena o han ejercido una actividad por cuenta propia.»

EMPIRIA. Revista de Metodología de Ciencias Sociales. N. ${ }^{\circ}$ 27, enero-abril, 2014, pp. 73-94. ISSN: 1139-5737 DOI 10.5944/empiria.27.10863 
- Porcentaje de población analfabeta y sin estudios: este indicador se obtiene de los datos sobre nivel de estudios (grados), para personas mayores de 16 años (edad de escolarización obligatoria) residentes en viviendas familiares, sumando los grados «personas analfabetas» $\mathrm{y}$ «personas sin estudios», según la definición del INE6 .

La vulnerabilidad se establece cuando el indicador es superior en un $50 \%$ a la media nacional. El valor medio nacional era en 2001 de $15,33 \%$, determinándose como índice de vulnerabilidad una tasa del 23,00\%.

- Porcentaje de personas en viviendas que no cuentan con aseo o baño:

Obtenido del Censo 2001, para personas que viven en viviendas principales que no tienen aseo o baño respecto al total, la vulnerabilidad se establece cuando el indicador es superior al doble del índice nacional. El valor nacional era en 2001 de 1,00\%, determinándose como índice de vulnerabilidad una tasa del 2,00\%. En 1991 se utilizaron: $2 \%$ sin aseo, $3 \%$ sin retrete, $5 \%$ sin baño o ducha en la vivienda (El Censo de 1991 los recogía de este modo. El de 2001 recoge agrupados sin aseo o baño).

Como ya advertimos, el Censo de Población y Vivienda constituye la única fuente con información extensiva e intensiva a nivel de sección censal, y en lo que se refiere a éste trabajo, la única que recoge los indicadores utilizados para definir las áreas vulnerables.

\subsection{Segunda fase: caracterización de los barrios vulnerables}

Una vez delimitadas las áreas vulnerables, el siguiente paso era el de proceder a la caracterización de dichas áreas, completando una información más exhaustiva sobre las mismas. Ello se hizo en un proceso secuencial que incluyó las siguientes acciones:

- Delimitación de las áreas sobre el plano de secciones censales:

A partir del listado de secciones censales vulnerables, se procedió a su delimitación sobre el plano de la ciudad, con el fin de realizar un primer análisis urbanístico, determinando continuidades, definiendo barreras y

${ }^{6}$ Se considera que una persona ha alcanzado un determinado nivel de instrucción cuando ha terminado y aprobado todos los cursos de ese nivel y está en condiciones, por tanto, de obtener el título o diploma correspondiente.

Analfabetos: Se considera que una persona es analfabeta cuando no sabe leer o escribir en ningún idioma,

Sin estudios: Personas que saben leer y escribir pero fueron menos de 5 años a la escuela. Aunque esta categoría es equivalente a los «estudios primarios incompletos» de la Clasificación Nacional de Educación (CNED), se ha optado por mantener la denominación para facilitar la comparación con los Censos anteriores.»

EMPIRIA. Revista de Metodología de Ciencias Sociales. N. ${ }^{\circ}$ 27, enero-abril, 2014, pp. 73-94. ISSN: 1139-5737 DOI 10.5944/empiria.27.10863 
realizando un primer análisis de la homogeneidad morfológica de los espacios. La delimitación de barrios se hizo sobre la cartografía más actualizada disponible, apoyándose en la consulta de publicaciones y estudios de la ciudad correspondiente, con lo que se contaba con una delimitación de áreas homogéneas previa al trabajo de campo.

- Contactos con los Ayuntamientos:

El listado de contactos y direcciones para cada ciudad se basó en los directorios aportados por la Federación Española de Municipios y Provincias (FEMP), el Ministerio de Fomento y la sección de Urbanismo del Instituto Juan de Herrera. Sobre el listado previo se realizó una primera comunicación informando sobre los objetivos del trabajo, pidiendo un contacto para coordinar las relaciones con el Ayuntamiento y solicitando la siguiente información: plano de secciones censales, cartografía 1:25.000 y 1:5.000 e información sobre el desarrollo de la ciudad.

Más tarde se envió un segundo fax, directamente dirigido al coordinador (o reiterando su designación), en el que se incluía el listado de secciones censales vulnerables correspondiente al municipio. En las primeras semanas del mes de Septiembre 2011 se confirmaron las citas, para realizar la entrevista y en su caso insistir en las peticiones de documentación. La información solicitada fue la siguiente:

1. Planeamiento, equipamiento y acciones previstas:

- Antigüedad y origen del ámbito.

- Figuras de planeamiento que le afecten y desarrollo de estas.

- Acciones de mejora sociourbanística en cualquier grado de desarrollo.

- Proyectos y acciones con ayudas o con ayudas solicitadas.

2. Percepción de los problemas.

- Accesibilidad.

- Infraestructuras.

- Calidad de la edificación.

- Calidad del espacio público.

- Percepción socioeconómica.

- Medio ambiente urbano.

Tan solo en tres de los municipios contactados, fue imposible realizar una entrevista u obtener información complementaria del Ayuntamiento (Portugalete, Sagunto y Telde) no pudiéndose disponer de plano de secciones censales del Censo de 1991 en Sagunto y Telde. Santa Cruz de Tenerife sí nos envió el plano de secciones censales, pero no se pudo realizar la entrevista, ni el

EMPIRIA. Revista de Metodología de Ciencias Sociales. N. ${ }^{\circ}$ 27, enero-abril, 2014, pp. 73-94. ISSN: 1139-5737 DOI 10.5944/empiria.27.10863 
trabajo de campo, con lo que tan solo se ha realizado una división orientativa de las áreas vulnerables. Por ello en nuestro catálogo solo se incluye la delimitación sobre el plano de secciones censales pero no existe ficha descriptiva del área.

- Entrevista con la administración municipal:

La entrevista con representantes de la administración municipal de la ciudad correspondiente permitió contrastar la información con la percepción y el conocimiento de las autoridades locales. Así, en ocasiones existían áreas vulnerables que no afloraron en el análisis estadístico, pero sí eran consideradas como tales por el municipio. A su vez la explotación del Censo nos dio áreas con parámetros vulnerables que no eran percibidas como tales por nuestros interlocutores.

La entrevista con la administración municipal nos aportó informaciones complementarias sobre la situación real de los barrios y la existencia o no de proyectos o programas de intervención en ellos, su grado de vulnerabilidad y la delimitación del barrio funcional.

\section{- Trabajo de campo:}

La visita a 88 ciudades españolas requirió de un esfuerzo logístico considerable, tanto por la selección y formación del equipo de trabajo, como por la realización de un calendario razonable que incluyera las citas previas que aseguraran el éxito del trabajo. Las visitas se realizaron durante el mes de Septiembre y la primera semana de Octubre del año 2011. Ese trabajo permitió incluir informaciones complementarias sobre la situación real de los barrios y la existencia o no de programas o proyectos de intervención en ellos, así como su grado de vulnerabilidad y la delimitación más ajustada del Barrio Vulnerable.

\subsection{Tercera fase: profundización en el análisis de la vulnerabilidad}

Si la vulnerabilidad es un concepto multidimensional y complejo, los indicadores que permitan acceder a un conocimiento más profundo de la misma, deben tener también ese carácter multidimensional. El estudio propuesto es una nueva oportunidad para mejorar el tratamiento de la información, pensando en la necesidad de establecer una línea de trabajo continuada, un análisis longitudinal de los barrios desfavorecidos que se puede prolongar en futuros censos. En este sentido, parece pertinente incorporar nuevos indicadores simples y sintéticos, de tal modo que permitan establecer un gradiente de barrios vulnerables en función de la intensidad del desfavorecimiento. Al respecto

EMPIRIA. Revista de Metodología de Ciencias Sociales. N. ${ }^{\circ}$ 27, enero-abril, 2014, pp. 73-94. ISSN: 1139-5737 DOI 10.5944/empiria.27.10863 
se aporta una propuesta de nuevas variables e indicadores sintéticos ${ }^{7}$, con un doble objetivo:

- Caracterización de esos espacios, desde una perspectiva demográfica, socioeconómica, residencial y de percepción subjetiva. Es decir, es preciso operacionalizar la multidimensionalidad del concepto de la vulnerabilidad en indicadores que respondan a esas dimensiones consideradas y explicitadas en el concepto de vulnerabilidad desarrollado en la primera parte del artículo.

- Establecer un gradiente de vulnerabilidad a partir de una serie de indicadores en el ámbito demográfico, socioeconómico, residencial y de percepción subjetiva, que permita priorizar intervenciones de carácter socio-urbanístico por parte de los poderes públicos competentes.

Los indicadores utilizados para medir el grado de vulnerabilidad en sus cuatro dimensiones son los siguientes:

- Vulnerabilidad socio-demográfica:

- Porcentaje de hogares unipersonales mayores de 64 años: hogares constituidos por una sola persona mayor de 64 años respecto al conjunto de los hogares.

- Índice de sobre-envejecimiento: personas mayores de 74 años respecto al total de la población.

- Índice de población extranjera en edad infantil: niños menores de 15 años de nacionalidad extranjera (salvo UE-15) respecto al total de niños menores de 15 años.

- Índice de extranjería 2001 y 2006: porcentaje de extranjeros respecto al total de población, exceptuando los naturales de la UE-15.

- Porcentaje de hogares monoparentales: hogares con un adulto (hombre o mujer) y uno o varios menores, respecto al conjunto de los hogares.

\section{- Vulnerabilidad socio-económica:}

- Tasa de desempleo: porcentaje de parados respecto al total de población activa.

- Tasa de desempleo juvenil: porcentaje de parados de 16 a 29 años respecto al total de activos de 16 a 29 años.

- Tasa de ocupados eventuales: porcentaje de ocupados que son trabajadores por cuenta ajena con carácter eventual, temporal... sobre el total de ocupados (variable situación profesional).

7 Los indicadores sintéticos recogen en una sola expresión numérica las diferentes dimensiones del concepto que se pretende utilizar para la medición y el análisis, de tal modo que se construye con la combinación de distintos indicadores «simples» $\mathrm{O}$ «unidimensionales», con la intención de recoger la multidimensionalidad del concepto, en este caso de la «vulnerabilidad urbana». En ese sentido, para la identificación de barrios vulnerables se han combinado indicadores de las cuatro dimensiones desplegadas.

EMPIRIA. Revista de Metodología de Ciencias Sociales. N. ${ }^{2}$ 27, enero-abril, 2014, pp. 73-94. ISSN: 1139-5737 DOI 10.5944/empiria.27.10863 
- Tasa de trabajadores no cualificados: porcentaje de trabajadores no cualificados respecto al total de ocupados (variable ocupación profesional a 1 dígito de la CNO94).

- Tasa de población sin estudios: porcentaje de población mayor de 16 años que no dispone de ninguna titulación académica (analfabetos y sin estudios).

\section{- Vulnerabilidad residencial:}

- Porcentaje de viviendas con una superficie útil menor a 31 metros cuadrados: viviendas familiares principales convencionales que tienen una superficie útil menor de 31 metros cuadrados respecto al total de viviendas familiares principales convencionales.

- Superficie media de la vivienda por ocupante: metros cuadrados por ocupante en las viviendas familiares principales convencionales.

- Porcentaje de viviendas sin servicio y aseo: viviendas familiares principales convencionales que no tienen servicio o aseo dentro de la vivienda respecto al total de viviendas familiares principales convencionales (variable problemas de la vivienda).

- Porcentaje de viviendas situadas en edificios en mal estado de conservación: viviendas familiares principales convencionales situadas en edificios en situación ruinosa o deficiente respecto al total de viviendas familiares principales convencionales.

- Porcentaje de viviendas situadas en edificios construidos antes de 1951: viviendas familiares principales convencionales situadas en edificios construidos antes de 1951 respecto al total de viviendas familiares principales convencionales.

\section{- Vulnerabilidad subjetiva:}

- Porcentaje de viviendas cuya persona de referencia considera que su vivienda esta afectada por ruidos exteriores.

- Porcentaje de viviendas cuya persona de referencia considera que su vivienda esta afectada por contaminación o malos olores provocados por la industria, el tráfico...

- Porcentaje de viviendas cuya persona de referencia considera que su lugar de residencia tiene malas comunicaciones.

- Porcentaje de viviendas cuya persona de referencia considera que su lugar de residencia tiene pocas zonas verdes en su proximidad (parques, jardines...).

- Porcentaje de viviendas cuya persona de referencia considera que su lugar de residencia esta afectado por un medio social donde la delincuencia y el vandalismo son un problema. 


\section{LOS RESULTADOS}

Los resultados del estudio depararon cierta sorpresa, pues mientras las variables utilizadas para la determinación de los barrios vulnerables habían mejorado considerablemente entre 1991 y 2001, el número de Barrios Vulnerables aumentó de forma muy significativa. En el estudio basado en el Censo de 1991, se localizaron 376 barrios vulnerables en 81 de las 116 ciudades analizadas; en el catálogo basado en los datos del Censo de 2001, se han delimitado 624 barrios vulnerables en 103 ciudades de las 139 estudiadas. Este aumento en el número de barrios, y el de la población que los habita (con un crecimiento de un 54\%) es muy superior al crecimiento de la población española entre 1991 y 2001, que fue de un $9 \%$.

El aumento de la población residente en barrios vulnerables no se ha producido de manera homogénea entre las distintas comunidades autónomas. Del total de los barrios delimitados, más del $75 \%$ de ellos y casi el $80 \%$ de la población vulnerable, se encuentran en las cuatro comunidades autónomas de mayor población (Andalucía, Cataluña, Madrid y Valencia). Aunque esta situación se daba ya en 1991, la concentración era menor (66\% de los barrios y $69 \%$ de la población). Andalucía es la Comunidad Autónoma con más población vulnerable, tanto en 1991 como en 2001, mientras que Canarias, que era la segunda con mayor población en 1991, ha pasado al cuarto lugar, quedando por detrás de Cataluña y la Comunidad de Madrid. La Comunidad Valenciana, cuarta en número de habitantes analizados queda detrás de Canarias, a pesar de ser ésta última la sexta en población analizada. El resto de autonomías tienen menos de 100.000 residentes en Barrios Vulnerables.

Como podemos ver en el gráfico 4.1., que recoge las comunidades autónomas en relación al incremento neto de población vulnerable y a la variación porcentual de ésta respecto a la población total de la comunidad autónoma, nos encontramos con que el incremento de la vulnerabilidad ha sido muy dispar entre comunidades autónomas. La Comunidad de Madrid y Cataluña encabezan de manera muy significativa este incremento, quedando relativamente atrás en su evolución Andalucía. En cualquier caso tan solo Galicia tenía un decrecimiento, tanto en población neta como en porcentaje de población que residía en un barrio vulnerable. 
Gráfico 4.1. Relación entre la variación de población residente en barrios vulnerables 1991-2001 y la variación del porcentaje de población vulnerable respecto a la población total de la Comunidad Autónoma 1991-2001

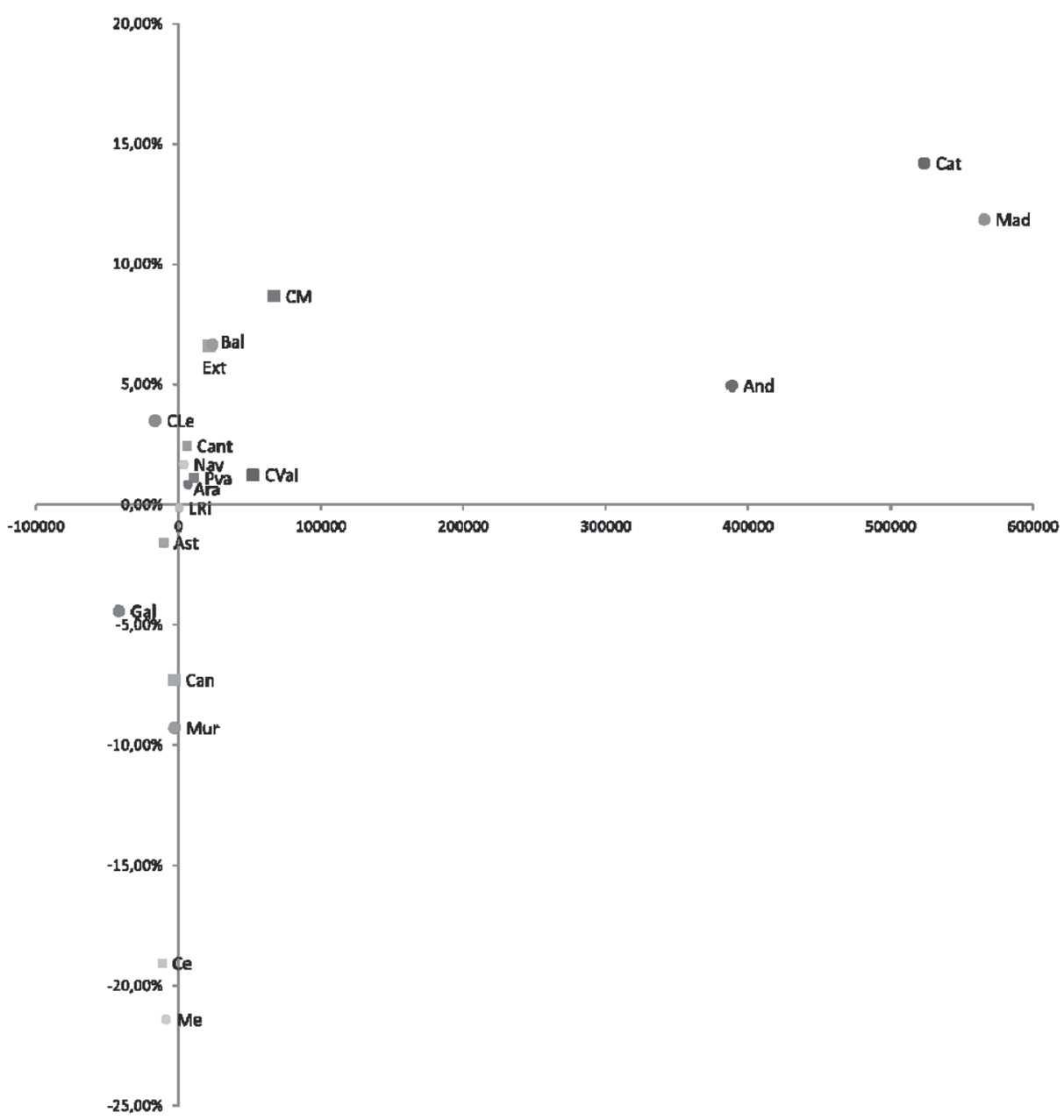

Fuente: Elaboración propia.

Leyenda: And, Andalucía; Ast, Principado de Asturias; Ara, Aragón; Bal, Balears (Illes); Can, Canarias; Cant, Cantabria; Cat, Cataluña; CLe, Castilla y León; CM, Castilla-La Mancha; CVal, Comunitat Valenciana; Ext, Extremadura; Gal; Galicia; LRi, La Rioja;, Mad Madrid (Comunidad de); Mur, Murcia (Región de); Nav, Navarra (Comunidad Foral de); Pva, País vasco; Ce, Ceuta; Me, Melilla:

Fuente: Elaboración propia. Datos. Censo 2001 y 1991

EMPIRIA. Revista de Metodología de Ciencias Sociales. N. . 27, enero-abril, 2014, pp. 73-94. ISSN: 1139-5737 DOI 10.5944/empiria.27.10863 
Si ya resulta chocante que en un marco de mejora generalizada de los indicadores de vulnerabilidad estudiados, se produzca un incremento tan significativo del número de barrios que se sitúan en una situación «peor» que la media nacional; resulta más sorprendente que sean la comunidades autónomas con un crecimiento más dinámico y con mejores ratios económicos aquellas en las que la vulnerabilidad ha aumentado de forma muy significativa sobre la media nacional. Si la pregunta de las causas de este crecimiento no puede ser contestada dentro del marco del trabajo realizado, no podemos dejar de realizarnos la pregunta sobre qué habrá ocurrido en el periodo 2001-2011, y qué está ocurriendo en estos tres años de crisis económica sostenida. Si el modelo urbano aplicado, si las políticas de redistribución aplicadas en tiempos de bonanza no permitieron reducir las diferencias en nuestras ciudades, qué podemos esperar de un periodo en el que estas políticas están a punto de desaparecer o diluirse en un marco de reducción o liquidación del Estado del Bienestar.

Mención aparte supone la descripción de las zonas en las que se ha producido un mayor crecimiento de la vulnerabilidad. En el gráfico 4.2. podemos ver que ésta se ha incrementado de manera muy significativa en los barrios periféricos, tanto en los barrios de bloque abierto y torres construidos en el periodo 1960-1975, en el que la corrupción franquista se alió con un sistema inmobiliario a la busca del mayor beneficio, como en las Parcelaciones Periféricas que fueron la forma de crecimiento coetánea al Ensanche, pero construida fuera de éste con el mínimo posible de normas para el alojamiento de la clase obrera que comenzó a llegar a las ciudades a principio del siglo XX. Es curioso que en este dudoso honor de ser los espacios en los que se incrementa de manera más significativa la vulnerabilidad urbana, se den la mano las dos periferias históricas que alojaron a las masas de clase obrera de nuestras ciudades. Al lector que no conozca gran parte de nuestros ensanches puede resultarle curioso que sean estos el segundo escalón en importancia en incremento de la vulnerabilidad urbana, pero tenemos que recordar que en ellos existen grandes áreas en que el tipo de vivienda se compone de un primer bloque de viviendas de mayor calidad y tamaño que dan a la calle, para tras de sí contener dos, tres, incluso más crujías de viviendas de menor calidad y con condiciones de habitabilidad muy reducidas. En cualquier caso hay que señalar que han desaparecido gran parte de las bolsas de infraviviendas que existían en 1991 (Parcelaciones Marginales) y se ha reducido relativamente el número de áreas periurbanas con viviendas y poblaciones con índices más precarios (probablemente absorbidas por el crecimiento urbano), También es significativa la pérdida de peso relativo de los Cascos Históricos de nuestras ciudades. 
Gráfico 4.2. Relación entre la variación de población residente en Barrios Vulnerables 1991-2001 y la variación del porcentaje de población vulnerable respecto a la población vulnerable total 1991-2001 según formas de crecimiento

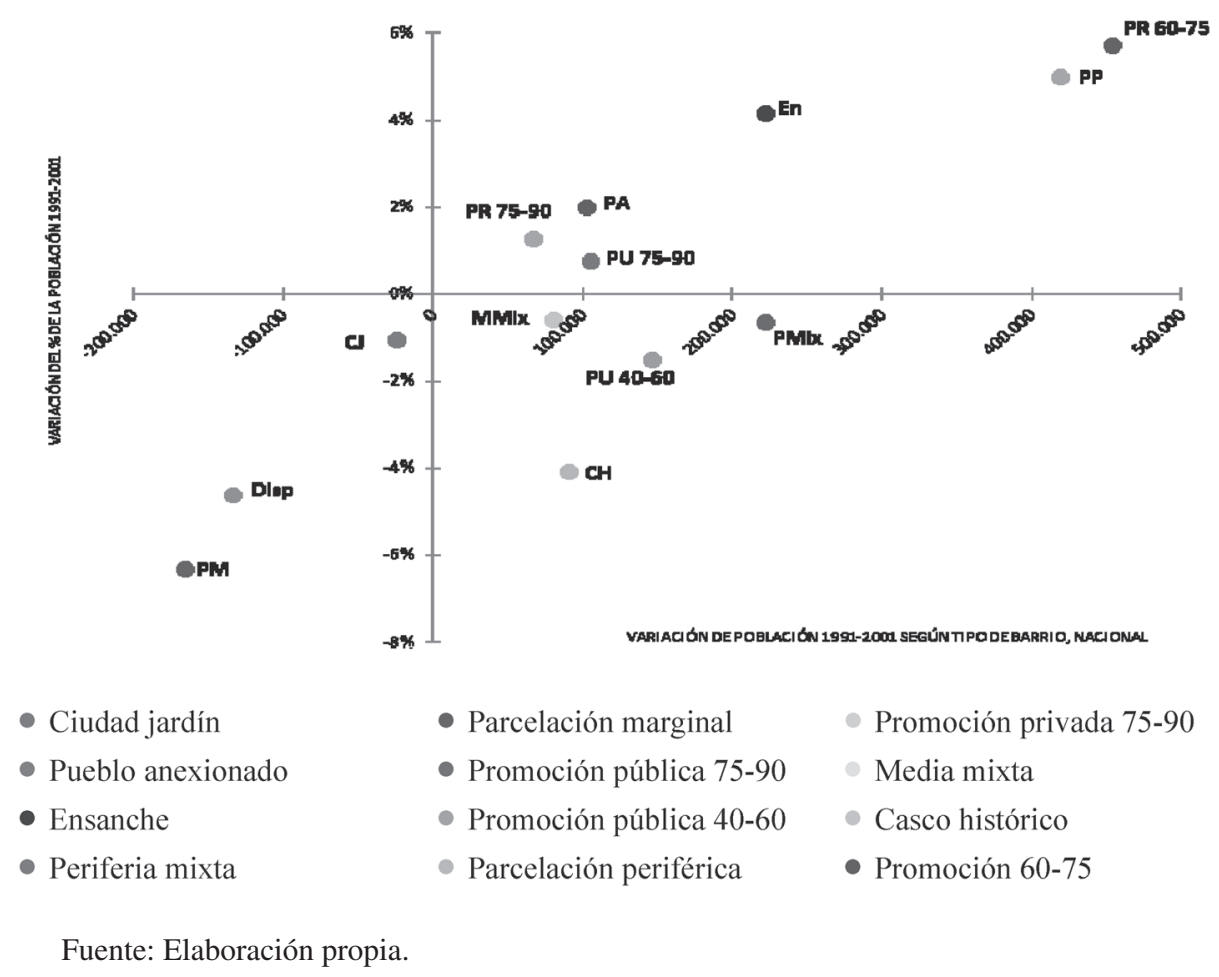

No es el momento de desgranar aquí los resultados del trabajo, ya que nuestro objetivo era el de presentar su metodología y la base teórica en la que se basa nuestro concepto de vulnerabilidad urbana, pero sí queremos reseñar que incluso pese a la relativa antigüedad de los datos utilizados (2001) el trabajo permite un análisis bastante consistente de la evolución de la vulnerabilidad de nuestras áreas urbanas, incluyendo la confirmación de la sospecha de que el modelo de desarrollo urbano y las políticas de redistribución empleadas en la etapa madura de la transición política española, no estaban garantizando la reducción de las diferencias sociales y espaciales existentes en nuestro país, y que cuanto mayor ha sido el crecimiento económico y urbano, mayor ha sido la evolución de la segregación social. Desgraciadamente, el hecho de que el Censo de 2011 no se haya realizado con la misma metodología y con el universo total de viviendas y habitantes, no nos va a permitir un análisis semejante al aquí presentado, aunque sí podría realizarse alguna aproximación con la información procedente de los padrones municipales.

EMPIRIA. Revista de Metodología de Ciencias Sociales. N. ${ }^{\circ}$ 27, enero-abril, 2014, pp. 73-94. ISSN: 1139-5737 DOI 10.5944/empiria.27.10863 
En cualquier caso, la difusión pública de los resultados permite a los investigadores y ciudadanos interesados en estudiar la evolución de sus ciudades, disponer de unas variables de comparación entre dos periodos de su historia y buscar cómo completar en cada caso el análisis realizado con la mejor información disponible. Quede nuestro catálogo como una información consistente para realizar estudios de la evolución de la vulnerabilidad en España, y como un método de trabajo que replicar, transformar o ampliar en función de la realidad espacial de cada ámbito y de los datos disponibles.

\section{BIBLIOGRAFÍA}

ALGUACIL, J. (2006): «Barrios desfavorecidos: un diagnóstico de la situación española», en V Informe FUHEM de políticas sociales: La exclusión social y Estado de Bienestar en España, Madrid, FUHEM-Icaria, pp. 155-168.

ARIAS, F. (Director) (2000): La desigualdad urbana en España, Madrid, Ministerio de Fomento.

BRUQUETAS, M.; MORENO, J.; WALLISER, A. (2005): La regeneración de barrios desfavorecidos, Documento de Trabajo 67, Madrid, Fundación Alternativas.

CASTEL, R. (1991): «La dinámica de los procesos de marginalización: de la vulnerabilidad a la exclusión». En Acevedo, M.; Volnovich, J. C.: El Espacio Institucional. Buenos Aires, Ed. Lugar, pp. 37-54

CASTEL, R. (1995) La métamorphoses de la question sociale, une chronique du salariat. Paris, Fayard,

EDIS; RENES, V.; ALGUACIL, J.; et al. (2000): Las condiciones de vida de la población pobre desde la perspectiva territorial. Pobreza y territorio, Madrid, FOESSA / Cáritas.

HERNÁNDEZ AJA, A. VÁZQUEZ ESPÍ, M. (2010) «Clasificación multicriterio: una regla simple para tratar problemas complejos» Sustentabilidad(es), no 2, pp. 192209, ISSN 0718-8846, ISSN 0718-8854.

HERNÁNDEZ AJA, A. (2007): «Áreas vulnerables en el centro de Madrid», Cuadernos de Investigación Urbanística, n. ${ }^{\circ}$ 53. Escuela Técnica Superior de Arquitectura. ISSN 1886-6654

http://www.aq.upm.es/Departamentos/Urbanismo/publicaciones/ciur53.html

HERNÁNDEZ AJA, A. (1997) «Catálogo De Barrios Vulnerables». Cuadernos de Investigación Urbanística, n. ${ }^{\circ} 19$. Universidad Politécnica de Madrid. Escuela Técnica Superior de Arquitectura. ISBN 84-87130-50-X http://www.aq.upm.es/Departamentos/Urbanismo/publicaciones/ciur19.html

LENOIR, R. (1974): Les exclus, un français sur dix. Paris, Éditions du Seuil

MOLINER, M. (2008): Diccionario de uso del español. Edición abreviada, Madrid, Gredos.

NACIONES UNIDAS. DEPARTAMENTO DE ASUNTOS ECONÓMICOS Y SOCIALES (2003): «Informe sobre la situación social del mundo 2003. Vulnerabilidad social: Fuentes y desafíos», Nueva York, United Nations Publications.

VV.AA. (2013): La vivienda en España en el siglo XXI. Diagnóstico del modelo residencial y propuestas para otra política de vivienda. Madrid, Fundación FOESSA.

EMPIRIA. Revista de Metodología de Ciencias Sociales. N. ${ }^{\circ}$ 27, enero-abril, 2014, pp. 73-94. ISSN: 1139-5737 DOI 10.5944/empiria.27.10863 